\title{
STEM-EDXS System for Atomic-Sensitivity Elemental Mapping
}

\author{
T.C. Lovejoy ${ }^{1}$, R.M. Stroud ${ }^{2}$, N.D. Bassim², G.J. Corbin ${ }^{1}$, N. Dellby ${ }^{1}$, W. Hahn ${ }^{3}$, P. Hrncirik ${ }^{1}$, M. \\ Falke $^{3}$, A. Kaeppel ${ }^{3}$, M. Rohde ${ }^{3}$, and O.L. Krivanek ${ }^{1}$ \\ 1. Nion Co., 11511 NE $118^{\text {th }}$ St. Kirkland, WA 98034, USA \\ 2. Materials Sci. and Tech. Division, Naval Research Laboratory, Washington, DC 20375, USA \\ 3. Bruker Nano GmbH, Am Studio 2D, 12489 Berlin, Germany
}

The Nion UltraSTEM aberration-corrected cold field emission scanning transmission electron microscope (AC-CFE-STEM) can focus a beam current of about $0.2 \mathrm{nA}$ into an atom-sized ( 1.5 $\AA$ large) electron probe at a primary energy of $60 \mathrm{keV}$ (and about $1 \mathrm{nA}$ at $200 \mathrm{keV}$ ). Using such an electron probe with an ultra-thin window silicon drift detector (SDD) of $30 \mathrm{~mm}^{2}(0.1 \mathrm{sr}$ solid angle) in a Mark I system at $60 \mathrm{keV}$ allowed energy dispersive X-ray spectroscopy (EDXS) to identify individual atoms while tracking their occasional hops to neighboring lattice sites [1]. Here we report on the design and performance of a Mark II STEM-EDXS system with about 10x higher efficiency, which allows single impurity atoms to be identified even when they are very mobile.

The new system uses a window-less SDD that is oval rather than round, and has an area of $100 \mathrm{~mm}^{2}$. Optimizing the shape of the objective lens polepiece and of the detector front end has allowed the detector to be brought to just $10.5 \mathrm{~mm}$ from the sample. Approximating the detector solid angle as $100 / 10.5^{2}$ gives $0.91 \mathrm{sr}$, accounting for the planar geometry of the detector gives $0.7 \mathrm{sr}$. Making the detector window-less provides a further significant gain in quantum efficiency, especially for lowenergy X-ray emission lines. The detector is mounted on a Nion UltraSTEM200 set up for voltages ranging from 40 to $200 \mathrm{kV}$, and operated at $60 \mathrm{kV}$ for this work.

To prevent sample contamination due to mobile hydrocarbons, or etching due to water vapor permeating through single O-rings and condensing on the sample, the microscope's column uses only metal seals and is bakeable to $140 \mathrm{C}$. The detector itself cannot be baked above $100 \mathrm{C}$, and it therefore needs to be removed from the column before baking. This is accomplished by having a gate valve that allows the detector to be isolated and removed without venting the column (Fig. 1). The internal (non-bakeable) volume of the detector is pumped with an ion pump and is maintained in the $10^{-7}$ torr range. The vacuum next to the sample is kept in the low $10^{-9}$ to high $10^{-10}$ torr range by a separate ion pump. When the detector is retracted, the two vacuums are separated by the gate valve; when the gate valve is opened and a motor drives the detector in, an O-ring sealing on the detector tube maintains the separation (Fig. 1). With the detector in the inserted position and cooled, the resultant samplelevel vacuum is high and clean enough for room temperature $60 \mathrm{keV}$ imaging of graphene to be possible without significant contamination or etching.

To reduce the impact of spurious system peaks without comprising system performance or the geometric solid angle with a collimator, the objective lens polepieces have been covered by thin copper caps, and $\mathrm{Be}-\mathrm{Cu}$ gimbals are used in the double-tilt sample holder. For a sample mounted on a $\mathrm{Cu}$ grid and clamped with a Be-Cu spring washer, there is primarily just one type of system peaks: $\mathrm{Cu}$, whose real concentration can be readily characterized by EELS. Figure 2 shows a spectrum from a $54 \mathrm{~nm}$ thick $\mathrm{NiO}$ calibration sample on a Molybdenum grid acquired at $60 \mathrm{keV}$ with $440 \mathrm{pA}$. Peaks from $\mathrm{Ni}, \mathrm{O}$, Moly, and $\mathrm{Cu}$ dominate; minor system peaks of $\mathrm{Ti}, \mathrm{Si}$, and $\mathrm{Al}$ remain. 
Figure 3 shows an example of detecting a mobile single atom of $\mathrm{Ca}$ on few-layer amorphous carbon, using tracking methodology as in [1], but with a significantly lower beam current (46pA), to mitigate the atom's greater mobility, and shorter time. There were $22 \mathrm{Ca}$ K-line counts in $35 \mathrm{~s}$ of tracking, during which the beam only spent about $1 \mathrm{sec}$ close enough to the Ca atom to ionize it. At $35 \mathrm{~s}$, the atom left the field of view of the tracking window. Similar count rates were recorded for other types of single atoms present in the sample, including silicon and sulphur. This demonstrates that the new system allows EDXS analysis to be carried out with single atom sensitivity for many elements in the periodic table, on real-world samples that change under the electron beam. See [2] for a practical application to heteroatom mapping in nanodiamond of extra-terrestrial origin.

\section{References}

1. T.C. Lovejoy et al., Appl. Phys. Lett. 100154101 (2012)

2. R.M. Stroud et al., (this meeting)

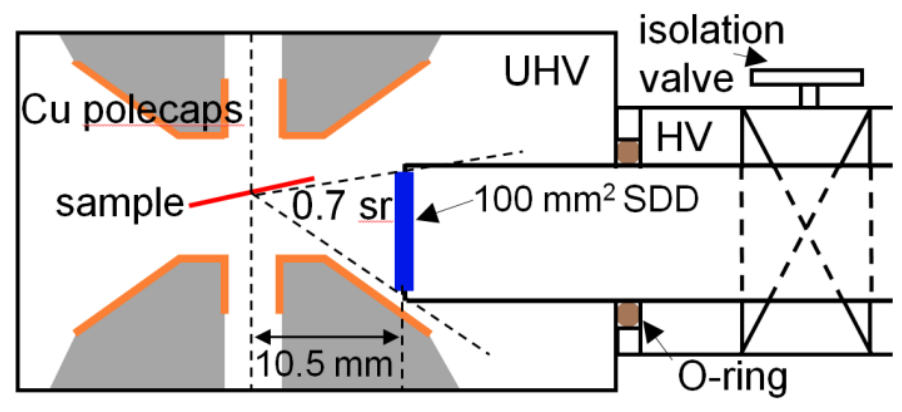

Fig. 1 Schematic drawing of $100 \mathrm{~mm}^{2}$ SDD chip $10.5 \mathrm{~mm}$ from sample showing copper polecaps over the polepieces, isolating $\mathrm{O}$ ring between the baked-ultra-clean sample vacuum (UHV) and non-baked detector vacuum (HV), isolating gate vale for when detector is retracted and approximate takeoff angles to detector extremes.

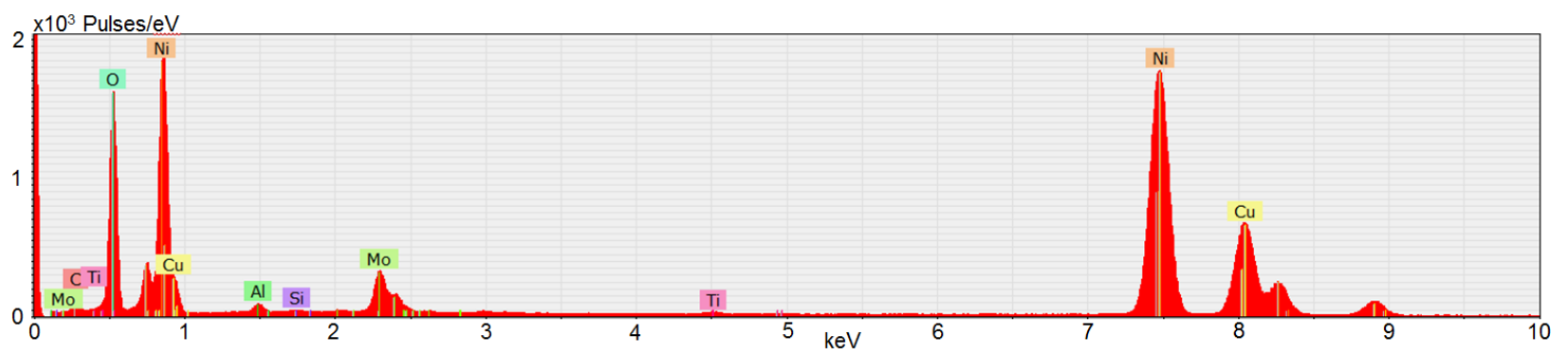

Fig. 2 Spectrum from $\mathrm{NiO}$ on Mo grid showing primarily $\mathrm{Cu}$ system peaks.
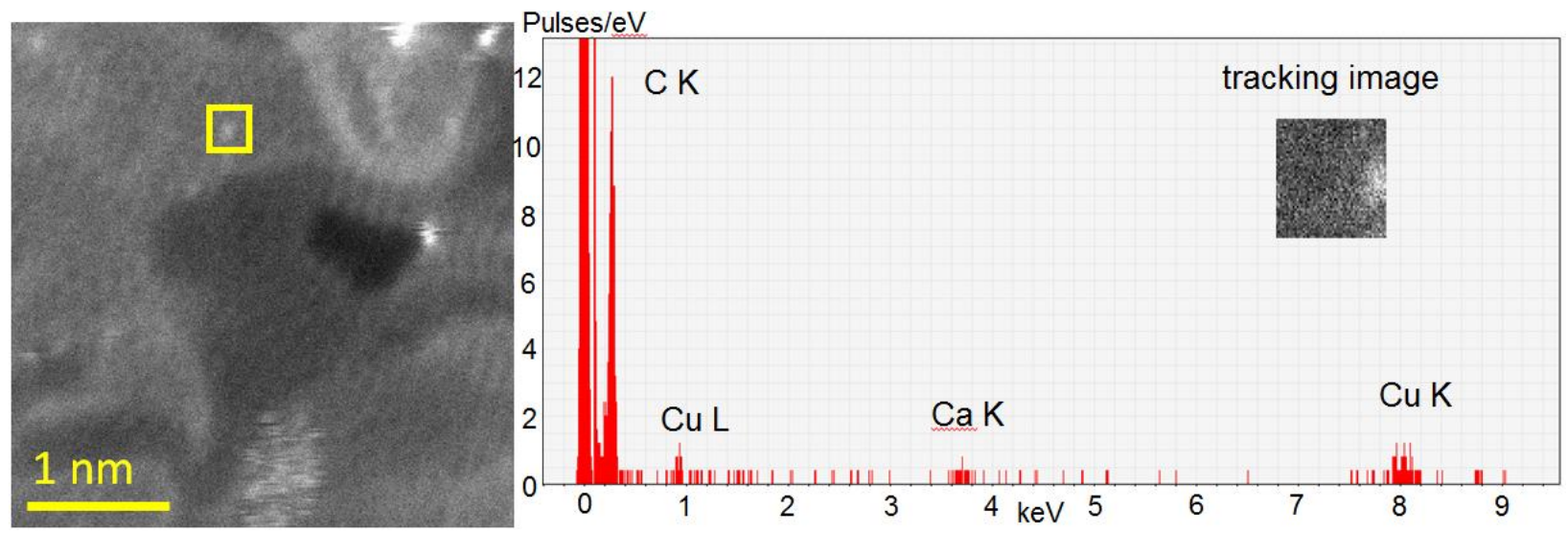

Fig. 3 (left) overview dark-field image of few-layer amorphous carbon showing several different species of isolated impurity atoms. The highlighted atom was tracked using the window shown (inset) to produce an X-ray spectrum (right) with $22 \mathrm{Ca} \mathrm{K}$-line counts in $35 \mathrm{~s}(60 \mathrm{kV}, 46 \mathrm{pA})$. 